\title{
Efficacy of extracorporeal membrane oxygenation as a bridge to lung transplantation
}

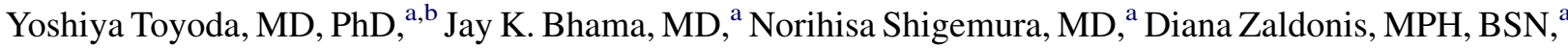 \\ Joseph Pilewski, MD, ${ }^{a}$ Maria Crespo, MD, ${ }^{a}$ and Christian Bermudez, MD ${ }^{a}$
}

\begin{abstract}
Background: Preoperative extracorporeal membrane oxygenation (ECMO) is a risk factor for poor outcome and currently considered a contraindication to lung transplantation. The lung allocation score system was introduced in May 2005 and prioritizes lung allocation to those with the greatest respiratory impairment. The purpose of this study is to determine whether ECMO as a bridge to lung transplantation is an acceptable option to support those in respiratory failure until donor lungs become available in the lung allocation score era.
\end{abstract}

Method: A retrospective review of 715 consecutive lung transplants performed between May 2005 and September 2011 was conducted using a prospectively collected institutional registry database. Twenty-four lung transplants $(3.4 \%)$ were performed in the 31 patients with attempted pretransplant ECMO; 7 patients who received ECMO patients did not survive or were deemed unfit for transplantation. These patients were compared with a control group of 691 patients who did not receive pretransplant ECMO.

Results: The duration of pretransplant ECMO was $171 \pm 242$ hours (median, 91 hours). Venovenous ECMO was used for respiratory failure in 15 patients, whereas venoarterial ECMO was used for circulatory collapse due to pulmonary hypertension in 9 patients. Patients in the retransplant ECMO group were younger ( $46 \pm 15$ years vs $57 \pm 14$ years, $P<.01$ ) compared with the control group, with no difference in recipient gender (male/female: $10 / 14$ vs $380 /$ 311 ), donor age ( $33 \pm 14$ years vs $36 \pm 15$ years), or donor gender (male/female: 10/14 vs 352/339). Emphysema was less common $(1,4 \%$ vs $260,38 \%, P<.01)$, and cystic fibrosis $(5,21 \%$ vs $72,10 \%, P=.09)$, redo lung transplant $(3,13 \%$ vs $28,4 \%, P=.08)$, and bronchiectasis $(2,8 \%$ vs $6,1 \%, P=.03)$ were more common in the pretransplant ECMO group. Patients in the pretransplant ECMO group had a significantly higher lung allocation score ( $87 \pm 9$ vs $44 \pm 15, P<.01)$. All patients in the pretransplant ECMO group underwent double lung transplants on pump (cardiopulmonary bypass/ECMO), and single lung transplants were performed in 171 patients (25\%) and pump was used in 243 patients (35\%) in the control group. The cardiopulmonary bypass time was longer in the pretransplant ECMO group ( $277 \pm 69$ minutes vs $225 \pm 89$ minutes, $P=.02$ ), with no difference in ischemic time ( $343 \pm 93$ minutes vs $330 \pm 98$ minutes, $P=.54$ ). Cadaveric lobar lung transplants were performed because of the urgency to overcome size mismatch with an oversized donor more frequently in $25 \%(\mathrm{n}=6$, no mortality with the longest follow-up at 6 years) of patients in the pretransplant ECMO group versus $0.3 \%(n=2)$ of patients in the control group $(P<.01)$. Post-transplant ECMO was used for primary graft dysfunction in 13 patients $(54 \%)$ in the pretransplant ECMO group and 41 patients $(6 \%)$ in the control group $(P<.01)$. The median hospital stay was 46 days in the pretransplant ECMO group versus 27 days in the control group $(P=.16)$. The actuarial survivals after lung transplants at $1,3,6,12$, and 24 months were $96 \%, 88 \%, 83 \%, 74 \%$, and $74 \%$, respectively, in the pretransplant ECMO group, and $97 \%, 94 \%, 90 \%, 83 \%$, and $74 \%$, respectively, in the control group $(P=.787)$.

Conclusions: Although the incidence of primary graft dysfunction requiring post-transplant ECMO is higher and the hospital stay is longer in patients receiving pretransplant ECMO, the graft survival is good (2-year survival, 74\%). ECMO is efficacious as a bridge to lung transplantation with good post-lung transplant outcomes. (J Thorac Cardiovasc Surg 2013;145:1065-71)

Extracorporeal membrane oxygenation (ECMO) has been used in clinical medicine for 40 years, but it remains a controversial therapy. ${ }^{1}$ ECMO has been used as a life-support tool for critically ill patients who can no longer survive

\footnotetext{
From the University of Pittsburgh, ${ }^{\mathrm{a}}$ Pittsburgh, Pa; and Temple University, ${ }^{\mathrm{b}}$ Philadelphia, Pa.

Disclosures: Authors have nothing to disclose with regard to commercial support. Read at the 92nd Annual Meeting of The American Association for Thoracic Surgery, San Francisco, California, April 28-May 2, 2012.

Received for publication May 4, 2012; revisions received Dec 4, 2012; accepted for publication Dec 18, 2012; available ahead of print Jan 18, 2013.
}

with optimum medical therapy, including maximal mechanical ventilator support, inhaled nitric oxide, and medications such as inotropes. Preoperative mechanical ventilation and hospitalization have been repeatedly shown

Address for reprints: Yoshiya Toyoda, MD, PhD, Division of Cardiovascular Surgery, Department of Surgery, Temple University School of Medicine, Suite 301 , Zone C, 3401 N Broad St, Philadelphia, PA 19140 (E-mail: yoshiya.toyoda@ tuhs.temple.edu) 0022-5223/\$36.00

Copyright (c) 2013 by The American Association for Thoracic Surgery http://dx.doi.org/10.1016/j.jtcvs.2012.12.067 


\section{Abbreviations and Acronyms \\ $\mathrm{ECMO}=$ extracorporeal membrane oxygenation \\ LAS = lung allocation score \\ UNOS $=$ United Network for Organ Sharing \\ $\mathrm{VA}=$ venoarterial \\ $\mathrm{VV}=$ venovenous}

to be significant risk factors for mortality in lung transplantation. ${ }^{2}$ Because patients receiving ECMO are hospitalized and almost always on mechanical ventilation, it has been controversial whether patients on ECMO should receive lung transplantation, and in reality, these patients have been frequently denied for listing or removed from the waitlist, resulting in pretransplant mortality. Mason and colleagues $^{3}$ analyzed United Network for Organ Sharing (UNOS) data from 1987 to 2008 and found that only 51 patients $(0.3 \%)$ in the United States were on ECMO preoperatively. The 1- and 2-year survivals were $50 \%$ and $45 \%$, respectively, for patients with pretransplant ECMO compared with $79 \%$ and $70 \%$, respectively, for unsupported patients. Therefore, ECMO has been considered a contraindication for lung transplantation in many centers because of the poor outcomes.

Before the introduction of the current lung allocation score (LAS) system in the United States in May 2005, ${ }^{4}$ lung allocation was primarily based on waiting time. The waiting time-based lung allocation favors patients well enough to wait the longest and does not favor critically ill patients who cannot wait for a prolonged period of time. ${ }^{5}$ Therefore, patients on ECMO might have to wait for a long time, and the outcomes of lung transplantation would be suboptimal because complications such as muscular deconditioning, infection, thromboembolism, bleeding, and poor nutrition could occur while waiting on ECMO. However, with the LAS system, critically ill patients who are in imminent danger of death and therefore in direst need of lung transplantation receive a high score and have priority of lung allocation. ${ }^{4,5}$ Patients on ECMO have a high $\mathrm{LAS},{ }^{3}$ possibly resulting in finding suitable donor lungs in a timely fashion, and therefore potentially leading to better outcomes.

We have recently reported our experience of pretransplant ECMO. ${ }^{6}$ However, the study included both lung and heart-lung transplant recipients and patients who were under various protocols from 1991 to 2010 . In addition to the change in the lung allocation in 2005, we have made several important changes in our protocols, including preservation solution of the donor lungs, intraoperative pulmonary protection, postoperative ventilator management, and immunosuppression from 1991 to $2003 .^{7,8}$ Therefore, the purpose of this study was to review the efficacy of ECMO as a bridge to lung transplantation, not including heart-lung transplantation, following the instrumentation of the current LAS system in 2005 in our current, standardized protocols at a single institution.

\section{PATIENTS AND METHODS Study Protocol}

The University of Pittsburgh Medical Center lung transplant evaluation and recipient research registry is approved by the University of Pittsburgh Institutional Review Board for the use of patient management, quality assurance reports, and clinical research. Data were prospectively collected into the Transplant Patient Management System. We performed a retrospective analysis of consecutive patients, from May 2005 to September 2011, who underwent lung transplant (primary and retransplantation). Data were obtained from the University of Pittsburgh Medical Center transplant database and patient charts. This study was approved by the Total Quality Council at the University of Pittsburgh Medical Center. The informed consent requirement was waived.

\section{Patient Selection}

ECMO was selectively used to support patients with advanced cardiopulmonary failure unresponsive to maximal medical therapy, such as mechanical ventilation support with $100 \%$ inspired oxygen fraction, positive end-expiratory pressure, and use of inhaled nitric oxide and inotropes. ECMO was considered for patients who presented a rapid deterioration of a chronic lung disease while on the waiting list or during the lung transplant evaluation process. Three patients on ECMO support for primary graft failure after the primary lung transplant with the absence of lung recovery were selectively considered for redo lung transplantation. We considered retransplantation in the context of primary graft dysfunction when other organ functions were intact. We excluded patients who did not meet standard criteria for lung transplant candidacy. Therefore, patients with other established organ dysfunctions, including renal failure, liver failure, major stroke, and sepsis, were denied. Patients who underwent lung transplantation without the use of pretransplant ECMO during the period analyzed served as a control group.

\section{Lung Transplant Protocols}

During the study period 2005 to 2011, standardized protocols were applied, which have been described. ${ }^{7,8}$ In summary, for donor lung procurement, a bolus injection of prostaglandin $\mathrm{E}_{1} 500 \mu \mathrm{g}$ was administered into the main pulmonary artery immediately before crossclamp. An additional $500 \mu \mathrm{g}$ of prostaglandin $\mathrm{E}_{1}$ and $50 \mathrm{mg}$ of nitroglycerin were added in the first bag of Perfadex (Vitrolife AB, Gothenburg, Germany). We administered $70 \mathrm{~mL} / \mathrm{kg}$ of Perfadex antegradely through the main pulmonary artery in the operative field and 1 liter of Perfadex for each lung retrogradely through the pulmonary veins at the back table. During the recipient surgery, $800 \mathrm{~mL}$ of cold blood with glutamate, aspartate, lidocaine, adenosine, nitroglycerin, verapamil, deferoxamine, ascorbic acid, dextrose, and insulin, as described previously, ${ }^{7}$ were given antegradely through the pulmonary artery after the bronchial anastomosis, and $800 \mathrm{~mL}$ of terminal warm blood with the same additives were given antegradely through the pulmonary artery before reperfusion to protect the allograft. Protective ventilatory management with low tidal volume $(6 \mathrm{~mL} / \mathrm{kg}$ of the donor body weight) and high positive end-expiratory pressure was used postoperatively. For immunosuppression, our standard immunosuppressive induction became alemtuzumab (Campath 1-H; Genzyme Corporation, Cambridge, Mass) in 2003, which was given intraoperatively. For maintenance immunosuppression, a triple drug regimen including tacrolimus, mycophenolate mofetil in half dose (750 mg twice daily), and minimized steroid (5 mg once daily) were used. For infection prophylaxis, valganciclovir was used for cytomegalovirus and voriconazole was used for fungus and yeast. 


\section{Extracorporeal Membrane Oxygenation Methodology}

Venovenous (VV) ECMO was the preferred method for respiratory failure in lung transplant candidates. Venoarterial (VA) ECMO was used when patients had cardiac failure in addition to respiratory failure. A Medtronic Carmeda (Medtronic Inc, Minneapolis, Minn) heparin-bonded Affinity oxygenator or QuadroxD oxygenator (Maquet Cardiopulmonary, Rastatt, Germany) was used in this study. Heparin (5000 units) was administered at the time of ECMO implantation and then titrated to maintain an activated clotting time of 140 to 160 seconds with VV ECMO and 180 to 200 seconds with VA ECMO.

\section{Data Analysis}

Statistical analysis was performed using the Statistical Package for the Social Sciences version 14.0 (SPSS Inc, Chicago, Ill). Continuous variables are shown as mean \pm standard deviation or median. The unpaired, 2-tailed $t$ test was used to compare independent continuous variables between the 2 groups. The Fisher exact test was used to determine whether there are nonrandom associations between 2 categoric variables. Kaplan-Meier analysis was used to calculate actuarial survival, and the log-rank test was used to test the null hypothesis that there is no difference in Kaplan-Meier survival curve between the groups.

\section{RESULTS \\ Cohort Characteristics}

The total cohort included 715 consecutive lung transplants. Of these, $24(3.4 \%)$ were performed for patients who were brought to the operating room already on pretransplant ECMO (pretransplant ECMO group), whereas 691 patients $(96.6 \%)$ did not receive ECMO support before lung transplant (control group). An additional 7 patients were placed on ECMO with the intent to perform transplantation, but for various reasons they did not undergo transplantation and died. Intraoperative insertion of ECMO was not considered as pretransplant ECMO. Of 24 patients, 15 required VV ECMO alone and 9 required VA ECMO. In VV ECMO $(n=15)$, the femoral vein and internal jugular vein were the most common cannulation sites $(\mathrm{n}=10)$, followed by dual-lumen cannula through the right internal jugular vein (Avalon Elite [Avalon Laboratories, LLC, Rancho Dominguez, Calif] $n=3$ ) and femoral vein-femoral vein $(n=2)$. In VA ECMO $(n=9)$, femoral vein-femoral artery $(\mathrm{n}=4)$, right atrium-ascending aorta $(\mathrm{n}=4)$, and internal jugular vein-femoral artery $(n=1)$ were the most common cannulation sites. The duration of pretransplant ECMO support in the pretransplant ECMO group was $171 \pm 242$ hours (range, 2-1104 hours). Superior vena cava obstruction due to $27 \mathrm{~F}$ dual-lumen cannula in the right internal jugular vein occurred in 1 patient, and the ECMO was converted to central VA ECMO with the right atrium to ascending aorta via the right thoracotomy. Three other patients who received right-atrium-ascending aorta central VA ECMO underwent their primary lung transplants and the ECMO was used as a bridge to retransplantation. Patients in the pretransplant ECMO group were significantly younger than patients in the control group $(P<.01)$. There were no differences in recipient gender, donor age, and donor gender between the groups. The LAS for the pretransplant ECMO group was significantly higher compared with the control group, with scores of $87 \pm 9$ and $44 \pm 15$, respectively $(P<.01)$ (Table 1$)$. Emphysema was less common $(4 \%)$ in the pretransplant ECMO group compared with $38 \%$ in the control group $(P<.01)$. Cystic fibrosis $(P=.09)$, bronchiectasis $(P=.03)$, and redo lung transplants $(P=.08)$ were more common in the pretransplant ECMO group (Table 2). Seven patients started on ECMO as a potential bridge to transplant did not undergo transplantation and died while on ECMO support. Therefore, the bridge to lung transplant success rate was $77 \%$. ECMO was not considered for patients who had multiple organ failure, resulting in deaths on the waiting list.

\section{Operative Characteristics}

All patients in the pretransplant ECMO group underwent double lung transplants $(24 / 24 ; 100 \%)$, whereas $75 \%$ (520/691) of the patients in the control group underwent double lung transplants $(P<.01)$. Pump (cardiopulmonary bypass or ECMO) was used in all patients in the pretransplant ECMO group, whereas only 35\% (243/691) of the patients required pump in the control group to perform lung transplants $(P<.01)$. Cardiopulmonary bypass time was longer for the pretransplant ECMO group $(277 \pm 69$ minutes) than for the control group $(225 \pm 89$ minutes $)$ $(P=.02)$. The ischemic time was similar between the groups

TABLE 1. Patient characteristics

\begin{tabular}{|c|c|c|c|}
\hline & $\begin{array}{l}\text { Pretransplant } \\
\text { ECMO }\end{array}$ & Control & $P$ value \\
\hline No. & $24(3.4 \%)$ & $691(96.6 \%)$ & - \\
\hline Recipient age (y) & $46 \pm 15$ & $57 \pm 14$ & $<.01$ \\
\hline Recipient gender & $\begin{array}{c}10 \text { men }(42 \%) / 14 \\
\text { women }(58 \%)\end{array}$ & $\begin{array}{c}380 \text { men }(55 \%) / 311 \\
\text { women }(45 \%)\end{array}$ & .216 \\
\hline Donor age (y) & $33 \pm 14$ & $36 \pm 15$ & .202 \\
\hline Donor gender & $\begin{array}{c}10 \text { men }(42 \%) / 14 \\
\text { women }(58 \%)\end{array}$ & $\begin{array}{c}352 \text { men }(51 \%) / 339 \\
\text { women }(49 \%)\end{array}$ & .412 \\
\hline LAS & $87 \pm 9$ & $44 \pm 15$ & $<.01$ \\
\hline Transplant type & & & $<.01$ \\
\hline Single lung & $0(0 \%)$ & $171(25 \%)$ & \\
\hline Double lung & $24(100 \%)$ & $520(75 \%)$ & \\
\hline $\begin{array}{l}\text { Cadaveric lobar } \\
\text { transplant }\end{array}$ & $6(25 \%)$ & $2(0.3 \%)$ & $<.01$ \\
\hline CPB/ECMO use & $24(100 \%)$ & $243(35 \%)$ & $<.01$ \\
\hline CPB time (min) & $277 \pm 69$ & $225 \pm 89$ & .02 \\
\hline $\begin{array}{l}\text { Ischemic time per } \\
\text { lung (min) }\end{array}$ & $343 \pm 93$ & $330 \pm 98$ & .54 \\
\hline $\begin{array}{l}\text { Use of post-transplant } \\
\text { ECMO }\end{array}$ & $13(54 \%)$ & $41(6 \%)$ & $<.01$ \\
\hline $\begin{array}{l}\text { Median hospital } \\
\text { stay (d) }\end{array}$ & 46 & 27 & .16 \\
\hline
\end{tabular}


TABLE 2. Diagnosis

\begin{tabular}{lccc}
\hline & $\begin{array}{c}\text { Pretransplant } \\
\text { ECMO }(\mathbf{N}=\mathbf{2 4})\end{array}$ & $\begin{array}{c}\text { Control } \\
\mathbf{N}=\mathbf{6 9 1}\end{array}$ & $\begin{array}{c}\boldsymbol{P} \\
\text { value }\end{array}$ \\
\hline COPD & $1(4 \%)$ & $260(38 \%)$ & $<.01$ \\
IPF/fibrosis & $8(33 \%)$ & $219(32 \%)$ & .827 \\
Cystic fibrosis & $5(21 \%)$ & $72(10 \%)$ & .091 \\
Scleroderma/MCTD/Wegener's & $3(13 \%)$ & $48(7 \%)$ & .241 \\
Bronchiectasis & $2(8 \%)$ & $6(1 \%)$ & .027 \\
Sarcoidosis & $1(4 \%)$ & $18(3 \%)$ & .482 \\
Primary pulmonary hypertension & $1(4 \%)$ & $11(2 \%)$ & .338 \\
Redo lung transplantation & $3(13 \%)$ & $28(4 \%)$ & .081 \\
Others & $0(0 \%)$ & $29(4 \%)$ & .618 \\
\hline$E C M O$, Extracorporeal membrane oxygenation; $C O P D$, chronic obstructive pulmo- \\
nary disease; $I P F$, idiopathic pulmonary fibrosis; $M C T D$, mixed connective tissue \\
disease.
\end{tabular}

$(343 \pm 93$ minutes in the pretransplant ECMO group vs $330 \pm 98$ minutes in the control group) $(P=.54)$ (Table 1$)$.

\section{Cadaveric Lobar Transplantation}

Cadaveric lobar lung transplantation was selectively performed to overcome size discrepancy. To minimize the waiting time, given the critical condition of patients on pretransplant ECMO, $25 \%$ of patients in the pretransplant ECMO group received cadaveric lobar transplantation versus $0.3 \%$ of patients in the control group $(P<.01)$. The characteristics and intraoperative and postoperative data of the 6 patients are shown in Table 3 .

\section{Postoperative Characteristics}

ECMO support was used postoperatively for primary graft dysfunction in $54 \%$ of patients in the pretransplant ECMO group and $6 \%$ of patients in the control group $(P<.01)$.
The median hospital stay for the pretransplant ECMO group was longer (46 days) than for the control group (27 days) $(P=.155)$. Patients in the pretransplant ECMO group who also required post-transplant ECMO for severe primary graft dysfunction received a significantly greater amount of blood transfusion (31.8 \pm 5.7 units) compared with patients in the pretransplant ECMO group who did not require posttransplant ECMO $(17.3 \pm 2.8$ units, $P=.04)$.

\section{Survival}

In the pretransplant ECMO group, 1 patient $(4 \%)$ died within 30 days and 3 patients $(13 \%)$ died within 90 days. In the control group, 24 patients $(3 \%)$ died within 30 days and 43 patients $(6 \%)$ died within 90 days. Twenty patients $(83 \%)$ in the pretransplant ECMO group were discharged from the hospital, and 649 patients $(91 \%)$ in the control group were discharged from the hospital. The actuarial survivals after lung transplantation at 1, 3,6, 12, and 24 months were $96 \%, 88 \%, 83 \%, 74 \%$, and $74 \%$, respectively, in the pretransplant ECMO group, and 97\%, 94\%, $90 \%, 83 \%$, and $74 \%$, respectively, in the control group $(P=.787$, Figure 1$)$. There was no statistical difference $(P=.544)$ in the overall actuarial survival after lung transplant for patients with pretransplant VV ECMO versus patients with VA ECMO, with survivals of $100 \%, 93 \%, 87 \%$, $77 \%$, and $77 \%$ at $1,3,6,12$, and 24 months, respectively, in the pretransplant VV ECMO group and $89 \%, 78 \%, 78 \%$, $65 \%$, and $65 \%$ at $1,3,6,12$, and 24 months, respectively, in the pretransplant VA ECMO group.

\section{DISCUSSION}

This study examines a large single-center experience in the use of pretransplant ECMO support as a bridge to

TABLE 3. Cadaveric lobar transplants in double lung transplantation for patients on pretransplant extracorporeal membrane oxygenation $(\mathrm{N}=6)$

\begin{tabular}{|c|c|c|c|c|c|c|}
\hline Patient & 1 & 2 & 3 & 4 & 5 & 6 \\
\hline Recipient age (y) & 36 & 54 & 54 & 28 & 29 & 56 \\
\hline Recipient gender & Male & Female & Female & Female & Male & Male \\
\hline Recipient height (cm) & 180 & 152 & 163 & 145 & 170 & 183 \\
\hline Donor age $(y)$ & 52 & 21 & 42 & 30 & 38 & 18 \\
\hline Donor gender & Female & Male & Female & Male & Female & Male \\
\hline Donor height $(\mathrm{cm})$ & 170 & 180 & 173 & 183 & 170 & 173 \\
\hline Diagnosis & Fibrosis & Sarcoidosis & MCTD & $\mathrm{CF}$ & $\mathrm{CF}$ & Wegener's \\
\hline LAS & 89 & 93 & 91 & 89 & 82 & 90 \\
\hline Lobar transplant & LLL & LLL & RLL & $\mathrm{RUL}+\mathrm{RML}$ & RUL & $\mathrm{RUL}+\mathrm{RLL}$ \\
\hline \multicolumn{7}{|l|}{ Pretransplant ECMO } \\
\hline Type & VA & VA & VV & VA & VV & VV \\
\hline Duration (h) & 2 & 13 & 290 & 352 & 124 & 96 \\
\hline CPB time (min) & 243 & 181 & 205 & 319 & 287 & 450 \\
\hline Ischemic time left/right (min) & $277 / 377$ & $406 / 311$ & $228 / 323$ & $354 / 260$ & $449 / 449$ & $530 / 367$ \\
\hline Post-transplant ECMO & No & No & No & No & No & yes \\
\hline Hospital stay (d) & 59 & 24 & 48 & 51 & 42 & 47 \\
\hline Graft status (d) & Alive (2476) & Alive (365) & Alive (333) & Alive (281) & Alive (228) & Alive (220) \\
\hline
\end{tabular}

$L A S$, Lung allocation score; $E C M O$, extracorporeal membrane oxygenation; $C P B$, cardiopulmonary bypass; $M C T D$, mixed connective tissue disease; $C F$, cystic fibrosis; $L L L$, left lower lobe; $R L L$, right lower lobe; $R U L$, right upper lobe; $R M L$, right middle lobe; $V A$, venoarterial; $V V$, venovenous. 


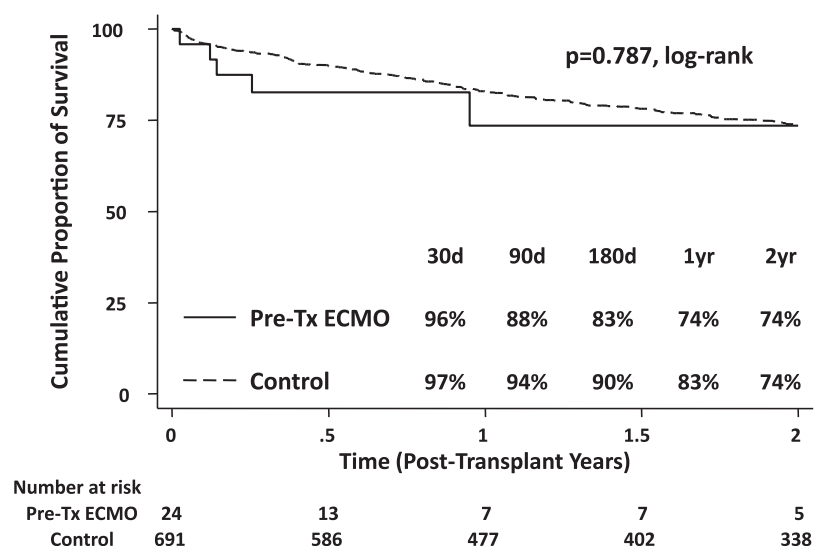

FIGURE 1. Kaplan-Meier graft survival after lung transplantation. $T x$, Transplantation; ECMO, extracorporeal membrane oxygenation.

lung transplant over a 6-year period (2005-2011) after the adoption of the LAS system in the United States. In our experience, $3.4 \%$ of the lung transplant recipients were on ECMO preoperatively, which is significantly higher than $0.3 \%$ in the UNOS data from 1987 to $2008 .^{3}$ The recipient age of patients with pretransplant ECMO was $46 \pm 15$ years, which was also significantly higher than the recipient age of $39 \pm 22$ years in the UNOS data. ${ }^{3}$ Our oldest recipient with pretransplant ECMO was aged 69 years. Of the 24 patients on pretransplant ECMO, $3(13 \%)$ had redo lung transplants and $2(8 \%)$ had scleroderma. These are known to be high-risk patients, who are often denied for lung transplantation by many centers even if they were without ECMO preoperatively. These data indicate that our program has a compassionate, aggressive posture giving critically ill patients on ECMO a chance of undergoing lung transplantation rather than denying them.

Since the implementation of the LAS system in May 2005 by the Organ Procurement and Transplantation Network, it has dramatically changed lung allocation from a system based purely on waiting time to an algorithm based on survival probability on the waiting list and after transplantation. The LAS has significantly reduced the waiting time and altered the distribution of lung diseases for which transplantation was performed on the basis of medical necessity. ${ }^{8}$ In our study, the mean waiting time was only 7 days (171 \pm 242 hours) for patients on pretransplant ECMO, all of whom had a high LAS (87 vs 44 in the control group; $P=.0001$, Table 1 ).

Although patients can tolerate ECMO with improved technology, ${ }^{1}$ the shorter the duration, the better the outcomes should be for obvious reasons, such as muscular deconditioning of the patients, blood loss or hemolysis, blood transfusions that can sensitize the patients, systemic or pulmonary thromboembolism, bleeding, infection, stroke, and so forth. One of the most common reasons why we turn down lung offers is size discrepancy, particularly for patients with small chest cavities. To overcome this issue, we have performed cadaveric lobar transplantation for 6 patients on pretransplant ECMO (Table 3). We determined which lobes were resected at the back table before implantation according to recipients' chest cavity and the size and quality of the donor lungs. All patients are surviving at the follow-up between 220 and 2476 days. Cadaveric lobar transplantation seems a viable option to shorten the waiting time and to achieve good outcomes especially for critically ill patients who cannot wait any longer, although long-term outcomes need to be determined.

Our patients with pretransplant ECMO had significantly higher rates of primary graft dysfunction requiring ECMO support postoperatively (54\% vs $6 \%$ ) and longer median hospital stay (46 vs 27 days, $P=.16$ ). This is in agreement with the findings previously reported. ${ }^{3,9}$ The reasons for the higher rates of primary graft dysfunction need to be clarified, but because of ECMO circulation and anticoagulation for the ECMO, patients are in a systemic inflammatory status and are coagulopathic, requiring more blood transfusions. We speculate these may be contributing factors. Patients who received pretransplant ECMO and required post-transplant ECMO for severe primary graft dysfunction received significantly greater amounts of blood transfusion ( $32 \pm 20$ units) compared with patients who received pretransplant ECMO but did not require post-transplant ECMO (17 \pm 9 units). Our data also suggest that experienced surgeons have less likelihood of requiring post-transplant ECMO (3 of 12;25\%) for severe primary graft dysfunction compared with less experienced surgeons (10 of $12 ; 83 \%$ ).

Despite higher rates of primary graft dysfunction and longer hospital stay, hospital mortality and 1- and 2-year survivals have not been adversely affected. The recipients with pretransplant ECMO showed similar $(P=.787)$ survivals compared with the no ECMO group, with pretransplant ECMO versus no ECMO survival of $96 \%$ versus $97 \%$ at 30 days, $83 \%$ versus $90 \%$ at 6 months, $74 \%$ versus $83 \%$ at 1 year, and $74 \%$ and $74 \%$ at 2 years, respectively (Figure 1). These survivals were better compared with UNOS data that included both pre-LAS and post-LAS era: The 30-day, 6-month, and 1- and 2-year survivals for patients with pretransplantation ECMO were $72 \%, 53 \%$, $50 \%$, and $45 \%$, respectively. ${ }^{3}$ A shorter waiting time for patients receiving pretransplant ECMO in the LAS era might contribute to better outcomes. In this study, we performed double lung transplantation for all of the patients with pretransplant ECMO, whereas in the UNOS data, only $57 \%$ of patients received double lung transplantation. Because the registry data show ${ }^{2}$ that survival is better with double lung transplantation versus single lung transplantation, this may be one of the reasons why our outcomes were better than those in the UNOS data. It is also possible that improved ECMO technology might have contributed to the better outcomes, both preoperatively and postoperatively. 
Patient selection clearly plays a key role in surgical treatments. During the study period from 2005 to 2011, we considered ECMO for all patients who were active on our institutional waiting list or who decompensated during the lung transplant evaluation process. However, the recipient age for patients with pretransplant ECMO was significantly lower than in the control group ( $46 \pm 15$ years vs $57 \pm 14$ years), suggesting that there was selection bias where age cutoff was lower for pretransplant ECMO consideration than the standard age cutoff for lung transplantation. We did not routinely consider ECMO use as a bridge to lung transplant in acute respiratory distress syndromes. Carefully selected patients were considered for lung retransplantation after severe allograft dysfunction, either chronic or acute after the initial lung transplantation. In our center, patients with other organ failure (eg, kidney and liver) were denied for redo lung transplantation. Therefore, when, for example, renal failure after ECMO developed in patients, they were in general not considered for lung transplantation.

The type of ECMO support used to bridge patients (VA or VV) to lung transplantation did not show a significant difference in overall survival, although the 1-year survival seemed higher at $77 \%$ with VV ECMO versus $65 \%$ with VA ECMO. At the University of Pittsburgh Medical Center, patients who were placed on VA ECMO had cardiac failure in addition to respiratory failure, whereas patients on VV ECMO had only respiratory failure. Therefore, patients who required VA ECMO might be sicker than patients who required VV ECMO. In addition, arterial cannulation, which is required for VA ECMO, can cause more complications, such as leg ischemia, arterial embolism (eg, stroke, ischemic gut), and bleeding complication with more intense anticoagulation. To avoid leg ischemia, we routinely inserted a distal perfusion cannula when a femoral artery was used for arterial cannulation. For VV ECMO, femoral vein to internal jugular vein was the most common cannulation site in this study. However, we and others have reported the use of a bicaval dual-lumen catheter as a bridge to lung transplantation. ${ }^{10-12}$ The potential advantage with this cannula is to allow patients to ambulate while waiting for lung transplantation so that muscular deconditioning can be avoided. In this study, 3 patients received a single dual-lumen catheter for VV ECMO; however, they also required mechanical ventilation for adequate oxygenation. The efficacy of this cannula and VV versus VA ECMO needs to be clarified through further experience.

\section{Study Limitations}

This single-center study is a small-scale, retrospective analysis with a short follow-up period. As such, the findings of this study are limited, and a prospective, multicenter study would provide more definitive conclusions.

\section{CONCLUSIONS}

After the implementation of the LAS system in 2005, ECMO as a bridge to lung transplantation is safe and efficacious to support patients until donor lungs become available. ECMO provides excellent survival when it is used in carefully selected patients and when lung transplants are performed by experienced surgeons, resulting in less blood loss.

The authors thank Yoshiko Toyoda at Cornell University, Mohammed A. Kashem, MD, PhD, and T. Sloane Guy, MD, at Temple University, and Victoria S. Dougherty, BA, for scientific and administrative assistance.

\section{References}

1. MacLaren G, Combes A, Bartlett RH. Contemporary extracorporeal membrane oxygenation for adult respiratory failure: life support in the new era. Intensive Care Med. 2012;38:210-20.

2. Christie JD, Edwards LB, Kucheryavaya AY, Benden C, Dobbels F, Kirk R, et al. The Registry of the International Society for Heart Lung Transplantation: Twenty-eighth Adult Lung and Heart-Lung Transplant Report-2011. J Heart and Lung Transplant. 2011;30:1104-22.

3. Mason D, Thuita L, Nowicki E, Murthy SC, Pettersson GB, Blackstone EH. Should lung transplantation be performed for patients on mechanical respiratory support? The US experience. J Thorac Cardiovasc Surg. 2010;139:765-73.

4. Egan TM, Murray S, Bustami RT, Shearon TH, McCullough KP, Edwards LB, et al. Development of the New Lung Allocation System in the United States. Am J Transplant. 2006;6:1212-27.

5. Egan TM, Kotloff RM. Pro/con debate: lung allocation should be based on medical urgency and transplant survival and not on waiting time. Chest. 2005;128:407-15.

6. Bermudez CA, Rocha RV, Zaldonis D, Bhama JK, Crespo MM, Shigemura N, et al. Extracorporeal membrane oxygenation as a bridge to lung transplant: midterm outcomes. Ann Thorac Surg. 2011;92:1226-32.

7. Toyoda Y, Thacker J, Santos R, Nguyen D, Bhama J, Bermudez C, et al. Longterm outcome of lung and heart-lung transplantation for idiopathic pulmonary arterial hypertension. Ann Thorac Surg. 2008;86:1116-22.

8. Shyu S, Dew MA, Pilewski JM, DeVito Dabbs AJ, Zaldonis DB, Studer SM, et al. Five-year outcomes with alemtuzumab induction after lung transplantation. J Heart Lung Transplant. 2011;30:743-54.

9. Kozower BD, Meyers BF, Smith MA, De Oliveira NC, Cassivi SD, Guthrie TJ, et al. The impact of the lung allocation score on short-term transplantation outcomes: a multicenter study. J Thorac Cardiovasc Surg. 2008;135:166-71.

10. Bermudez CA, Rocha RV, Sappington PL, Toyoda Y, Murray HN, Boujoukos AJ. Initial experience with single cannulation for venovenous extracorporeal oxygenation in adults. Ann Thorac Surg. 2010;90:991-5.

11. Fuehner T, Kuehn C, Hadem J, Wiesner O, Gottlieb J, Tudorache I, et al. Extracorporeal membrane oxygenation in awake patients as bridge to lung transplantation. Am J Respir Crit Care Med. 2012;185:762-8.

12. Olsson KM, Simon A, Strueber M, Hadem J, Wiesner O, Gottlieb J, et al. Extracorporeal membrane oxygenation in nonintubated patients as bridge to lung transplantation. Am J Transplant. 2010;10:2173-8.

\section{Discussion}

Dr Charles Hoopes (Lexington, Ky). Yoshi, let me start by commending you and your former colleagues at the University of Pittsburgh for your interest in a complex and difficult patient group. I think you are correct that historically these patients were not listed for transplant or, if they were listed, frequently died on the ventilator secondary to a failure of mechanical ventilation.

However, having said this and sharing with you a common experience and belief that there is in fact a role for mechanical circulatory support technologies in this patient group, it would also be fair to say that ours is a minority view and it is worth at least noting 
the legitimacy of some of these well-founded criticisms that are available in your own database.

As your retrospective study demonstrates, more than half of the patients undergoing transplantation from an ECMO bridge required ongoing extracorporeal support for primary graft dysfunction, and there is generally little debate that early allograft dysfunction is a predictor of early mortality and late allograft morbidity. It is clearly a predictor of prolonged postoperative courses as was demonstrated by your institutional study, where the average was 46 days for the bridging group as opposed to 27 days for those without a bridge, and it is fair to say that this is associated with a significant increase in health care costs.

In addition, as you noted, 6 of the 24 patients undergoing transplantation from ECMO received cadaveric lobar transplants because of donor-recipient size mismatches and the understandable need to limit the time of support on ECMO. So for those of us who may be cynics in the audience, it would be easy to view ECMO bridging as an expensive technology deployed in highrisk patients who subsequently receive high-risk allografts, albeit-and our experience has been the same-with comparable outcomes at 1 and 2 years.

However, putting the study in that context, let me ask you 1 question with basically 3 simple parts. First, from a philosophical standpoint, do you think that ECMO should be deployed as salvage in these patients awaiting transplantation, or should mechanical circulatory support in lung transplantation follow the same paradigm we currently follow in cardiac transplantation? In short, shouldn't ECMO take an unstable patient and create a lower-risk patient capable of a lower-risk transplant? In relation to that comment, if we are going to continue using ECMO bridging as salvage therapy for medically refractory patients failing mechanical ventilation, should we not consider once again the use of regionalization of donor organs rather than the traditional system of local centers first so as to prioritize organs to unstable patients on ECMO? And finally from a purely philosophical standpoint, do you see ECMO bridging as an evolving standard of care or should public health policy make this application of technology available only at a limited number of experienced referral centers?

Again, congratulations on dealing with this complex patient population, and I look forward to your comments.

Dr Toyoda. Let me comment on the frequency of postoperative ECMO use. I must admit we had a lower threshold to keep the ECMO going post-transplant because the patient was already cannulated. As you know, some centers use intraoperative ECMO to perform lung transplants, and they routinely keep the ECMO postoperatively. In this study, we were able to wean the ECMO early postoperatively in 4 patients, so ECMO might not be necessary for these 4 patients. Having said that, we still had approximately $30 \%$ to $40 \%$ of patients who required ECMO.

In terms of salvage, at the University of Pittsburgh we used ECMO as salvage in this study. I think that is okay, because our study shows a 74\% 2-year survival rate for the patients who otherwise would surely die then. We surgeons want to use our abilities to save patients' lives, so I think this is okay.

Regarding regionalization, I agree with you. For the sick patients, I think regionalization would be better than local center first policy. So we should review our lung allocation strategy.
In terms of centers, I am not so sure we should limit to certain centers, because the surgeon's experience is more important. Even in this study, an experienced surgeon who had performed more than 100 lung transplants had a better outcome in postoperative ECMO use and bleeding, and shorter warm ischemic time, hospital stay, and survival. So I don't think we should limit centers.

Dr Craig Smith (New York, NY). Dr Toyoda, I am looking for ways to make these data look better. Have you compared the outcomes in this group on ECMO with the patients in the other group who required cardiopulmonary bypass for double lungs?

Dr Toyoda. We did not analyze that, but that is a good idea and we will do it.

Dr R. Duane Davis (Durham, NC). Congratulations on an excellent series in obviously a difficult cohort of patients. I am going to follow-up what Dr Hoopes has said. Shouldn't we start thinking about this as a way of improving the candidacy of a lung transplant recipient, instead of just getting them to transplant, make them better? You had a 45-day length of stay. One of the things that we have seen is we got away from doing bedbound support with ECMO and started focusing on ambulatory ECMO. Then they start actually behaving like any other lung transplant recipient. The concept that has actually come out of Germany in terms of going preferentially to ECMO compared with ventilation and all the complications you buy with that type of strategy certainly seem to make a better recipient and outcome.

I would also be cautious to say that this should be widespread to any center. If you look at the UNOS data set and say what are the outcomes on all-comers for ECMO, they still are not really in an acceptable range. At single centers that have a focus on this, the outcomes can be acceptable, but you cannot say that they are the same. If you are trying to say the $74 \%$ in yours is exactly the same as your all-comers, that is just a statistical fluke of a low number of patients. Higher risk, you are going to have less good outcomes.

Dr Matthias Loebe (Houston, Tex). I have 2 quick questions. Can you expand on how many patients were put on ECMO in your institution who did not reach the point to be transplant candidates and then did not undergo transplantation? In other words, what is your selection from a patient on ECMO to undergo transplantation?

Can you comment on the lung allocation strategies? You mentioned repeatedly the fact that a LAS at this point does not get any extra points for being on mechanical support. The opposite is true that if you are put on mechanical support, pulmonary pressure goes down, or even if you are extubated, that will give you a certain disadvantage in organ allocation, as you know. So do you have any suggestions how to deal with this problem?

Dr Toyoda. During this time period we put an additional 7 patients on ECMO, but they did not reach lung transplantation. So, the success bridge rate is approximately $77 \%$. But of those 7 patients, our decision was wrong in probably 3 , because patients had multiorgan failure at the time of ECMO. So the real success rate may be $86 \%$ or so.

In terms of lung allocation, once a patient is on ECMO, we should consider that fraction of expired oxygen is $100 \%$ for those patients. ECMO is providing $100 \%$ oxygenation. So even if patients are extubated, we should probably give them a high score. 